\title{
RuleBase: Model Checking at IBM
}

\author{
I. Beer, S. Ben-David, C. Eisner, D. Geist, L. Gluhovsky, T. Heyman, \\ A. Landver, P. Paanah, Y. Rodeh, G. Ronin, Y. Wolfsthal \\ IBM Haifa Research Laboratory, Haifa, Israel \\ contact: beer@vnet.ibm.com
}

\begin{abstract}
RuleBase is a symbolic model checking tool, developed by the IBM Haifa Research Laboratory. It is the result of four years of experience in practical formal verification of hardware which, we believe, has been a key factor in bringing the tool to its current level of maturity. Our experience shows that after a short training period, designers can operate the tool independently and achieve impressive results. We present the tool and summarize our development and usage experience, focusing on some work done during 1996.
\end{abstract}

\section{Introduction}

RuleBase is a symbolic model checking tool, developed by the IBM Haifa Research Laboratory with the intent of bridging the formal verification usability gap. It is the result of four years of experience in practical formal verification of hardware and now offers usability, capacity and robustness features that make it an industrial-strength formal verification tool. In this note we present the tool, including several unique features, and summarize our usage experience.

RuleBase uses an enhanced version of SMV [12] as its verification engine, employing CTL model checking [5]. Much of the RuleBase development effort has been put into various techniques to address the state-explosion problem, thereby increasing the capacity of the core SMV engine and enabling verification of industrial designs. In its current state, RuleBase can verify design partitions consisting of up to 300 latches of control logic after reduction. This capacity, coupled with automatic reduction as a preprocessing phase, supports the verification of relatively large models. In pre-reduction terms, the largest unit verified by RuleBase has a few thousands of state-variables of control logic.

To make RuleBase an industrial tool, significant development effort has been made in several areas: First, since the temporal logic CTL is not an easy language for specification by non-experts, RuleBase has its own language - Sugar - built on top of CTL, which makes specification easier. Second, RuleBase supports standard, commonly used hardware description languages such as VHDL and Verilog, and operates within various design environments (e.g. Synopsys and Compass). Third, debugging tools are provided to aid in the analysis of verification results. Fourth, RuleBase is highly automated in every aspect of the verification process, particularly in HDL translation and design size reduction. Finally, RuleBase features a graphical user interface for interactive control of the verification process.

Environment models are written in the RuleBase language, a dialect of SMV which supports multiple environments (for instance, a read-only environment, a write-only 
environment and a read-write environment). Environment management is a key issue in the RuleBase methodology, facilitating enhanced capacity and usability of the tool.

\section{Prime Features of RuleBase}

\subsection{Withstanding Size Problems}

Symbolic model checking, introduced in [12], addresses the state explosion problem by using BDDs. While this has been a major advance and made model checking a useful tool for real hardware designs, the size problem is far from being solved. Much effort has been put into RuleBase in order to withstand the state explosion problem. Some of the methods are described below:

Automatic, Per-Formula Reduction. Usually, a formula is influenced by only part of the design, while other parts are irrelevant to its truth or falsity. For example, if the formula verifies a property of one design output, only this output and its input cone of logic are necessary. RuleBase identifies and removes unnecessary parts.

Efficient BDD Algorithms. RuleBase employs a variety of techniques and algorithms for handling BDD size problems. It uses variations of the dynamic reordering algorithm described in [14]. Additionally, RuleBase employs techniques of keeping the transition relation partitioned, as presented in [4] and [10]. Also, it combines BFS and DFS to maintain small BDD sizes during reachability analysis, following [13].

Checking Safety Formulas On-The-Fly. Formulas belonging to a subset of the CTL logic can be verified while traversing the reachable state space, without the need for the full model-checking algorithm. Several works $[6,11]$ used this method for formulas of the form $A G(p)$, where $p$ is a Boolean formula containing no temporal operators. RuleBase can check several safety formulas at a time, producing counter-examples to those formulas which fail without building the full transition relation, and continuing to check the rest.

\subsection{Sugar - RuleBase Specification Language}

RuleBase provides a specification language - Sugar - for hardware designers who are not CTL [5] experts, which allows them to read and write specifications easily. Sugar is built on top of CTL and includes a few additional language constructs. Some of the Sugar constructs are described below.

Within. Experience shows that many design behaviors are repetitive, where a basic transaction occurs again and again, and properties are interesting only within the boundaries of a single transaction. The within operator addresses this issue. Its syntax is "within( start, end )(Sugar-formula)", where start and end are Boolean expressions. The meaning is "check the Sugar formula only in time intervals beginning with start and ending with end". For example, "AG within( start, end )( $A G$ (request $\rightarrow A F$ acknowledge))" means: "in all time intervals delimited by start and $e n d$, a request must be followed by an acknowledge".

Next-Event. The operator "next_event $(p)(q)$ " has the following meaning: the next time that $p$ occurs, $q$ will occur. For example, "AG( (request \& requester=high_priority) 
-> next_event(grant)(granted=high_priority) )" means: "if there is a request from a high-priority device, then the next time there is a grant, the higher priority device is the one granted". Similarly "next_event $(p)[n](q)$ " means that $q$ must occur the $n t h$ time that $p$ occurs. For example: "AG( request $->$ next_event(data)[4](last_data) )" means: "last_data should be asserted together with the fourth data after a request". The CTL equivalent of this formula is: "AG ( request $->$ !E[!data $U$ (data \& EX El!data U (data \& EX E[!data U (data \& EX E[!data U (data \& !last_data ) ]) ]) ]) ])".

Strong and Weak Operators. Most Sugar operators have two forms: strong and weak. The strong form requires an event to happen eventually (liveness property), while the weak form only states that a bad event cannot happen (safety property). An operator becomes strong by appending a '!' to its name. For example, "next-event! $(p)[3](q)$ " (strong version) states that $p$ must happen three times and then $q$ must hold, while "next-event $(p)[3](q)$ " (weak version) states that if $p$ happens three times then $q$ must hold.

\subsection{Debugging Aids}

RuleBase has various tools to support the analysis of the model at hand as well as the results of the verification process. Some of these tools are described below.

Timing Diagrams. RuleBase presents counter-examples and witnesses (see below) as timing diagrams, making the debugging process similar to the one employed in traditional simulation-based verification.

Witness and Vacuity. When a formula passes successfully, RuleBase tries to produce a witness: an execution trace that demonstrates a non-vacuous path on which the formula holds. Analyzing a witness may help to discover unexpected behaviors resulting from wrong environment modeling or wrongly-formulated rules [3]. Also, the inability to produce a witness serves to indicate that the formula has passed vacuously.

Reduction Analyzer. RuleBase provides a detailed description and explanation of the reduction process, which users can call upon to explore, debug and control the elimination of state variables. This is specifically useful when experimenting with alternate reductions, of which the full effect is not clear to the user.

\section{Experience}

RuleBase has been used in various verification projects throughout its development. The following is a partial list of the units which were formally verified using RuleBase.

PCI bus bridges, including PCI-to-ISA, PCI-to-VESA and PCI-to-PCI bridges [1]. For each bridge, several dozens of PCI rules were written and verified. These included the full PCI specification as well as many implementation-level rules (e.g. performance). The formal verification of these units has revealed nearly 200 bugs, including several deadlocks. Three of the chips were fully functional at first silicon realization; the other two were fully functional at second silicon realization. A summary of using RuleBase in the verification of these designs can be found in [1].

An on-line L2-cache for a PowerPC processor. In this unit, formal verification has focused on three key blocks, namely the Processor Interface Unit, the System Interface 
Unit and the Cache Control Unit. Near 70 bugs were detected by RuleBase in this unit, which is a significant fraction of the design bugs found altogether with simulation.

Two cache coherency protocols. A 3-level directory-based MESI cache coherence protocol [9] and a distributed shared-memory MESI cache coherence protocol linking a number of tightly-coupled multi-processors [7] were modelled and verified. The formal verification effort revealed subtle specification bugs and omissions which would almost certainly not have been found using simulation.

Bus interface and cache control logic of a $\mathrm{AS400}$ processor. In a very recent project, IBM design engineers have found 65 bugs in the bus interface unit and cache control logic of an AS/400 processor, making a significant impact on the verification of this processor. Further information on this project is found in [8].

\section{References}

1. I. Beer, S. Ben-David, C. Eisner, Y. Engel, R. Gewirtzman, and A. Landver, "Establishing PCI Compliance using Formal Verification: a Case Study", Intl. Phoenix Conf. on Comp. and Comm. 1995.

2. I. Beer, S. Ben-David, C. Eisner and A. Landver, "RuleBase: An Industry-Oriented Formal Verification Tool", Proceedings of the Design Automation Conference, DAC'96.

3. I. Beer, S. Ben-David, C. Eisner and Y. Rodeh, "Efficient Detection of Vacuity in ACTL Formulas", this issue.

4. J. Burch, E. Clark and D. Long, "Representing Circuits More Efficiently in Symbolic Model Checking", DAC'91, pp. 403-407.

5. E. Clarke and E.A. Emerson, "Design and Synthesis of Synchronization Skeletons using Branching Time Temporal Logic", in proc. Workshop on Logics of Programs, LNCS 131, pp. 52-71, 1981 .

6. A. Eiriksson and K. McMillan, "Using Formal Verification/Analysis Methods on the Critical Path in System Design: A Case Study", CAV'95, LNCS 939, pp.367-380.

7. C. Eisner, "CC/NUMA Formal Verification Project - Summary and Report", IBM Internal Memorandum, 1995.

8. C. Eisner, "AS/400 SCU Formal Verification - Interim Report", IBM Internal Memorandum, 1997.

9. C. Eisner, G. Shurek, G. Meil, R. Raghavan, "CCP (Cache Coherence Protocol) Formal Verification Project Distributed Shared Memory Cache Coherence Protocol - Summary and Report", IBM Internal Memorandum, 1994.

10. D. Geist and I. Beer, "Efficient Model Checking by Automated Ordering of Transition Relation Partitions", CAV'94, LNCS 818, pp. 299-310.

11. D. Long, "Model Checking, Abstraction and Compositional Verification", Ph.D. Thesis, CMU, 1993.

12. K. McMillan, "Symbolic Model Checking", Kluwer Academic Publishers, 1993.

13. K. Ravi and F. Somenzi, "High-Density Reachability Analysis", Proceedings of the IEEE/ACM International Conference on Computer-Aided Design (ICCAD95), November 1995, San Jose, pp. 154-158.

14. R. Rudell, "Dynamic Variable Ordering for Ordered Binary Decision Diagrams", ICCAD'93, pp. 42-47. 